\title{
Evolut Self-Expanding Transcatheter Aortic Valve Replacement in Patients with Extremely Horizontal Aorta (Aortic Root Angle $\geq \mathbf{7 0}^{\circ}$ ) Technical Aspects and Clinical Outcomes
}

\author{
Umihiko Kaneko, ${ }^{1}$ MD, Daisuke Hachinohe, ${ }^{1}$ MD, Ken Kobayashi, ${ }^{1}$ MD, Hidemasa Shitan, ${ }^{1}$ MD, \\ Keijiro Mitsube, ${ }^{2}$ MD, Azusa Furugen, ${ }^{1}$ MD, Takeshi Kawamura, ${ }^{3}$ MD, \\ Ryuji Koshima, ${ }^{2}$ MD and Tsutomu Fujita, ${ }^{1}$ MD
}

\begin{abstract}
Summary
Because of its rigidity and non-steerability, the presence of a horizontal aortic root poses a major anatomical issue during transcatheter aortic valve replacement (TAVR) with Evolut self-expanding valve. Previous studies have elucidated the difficulties of coaxial implantation of the self-expanding valve in patients with horizontal aorta, often resulting in increased complications and a lower device success rate. To date, most patients with extremely horizontal aorta (aortic root angle $\geq 70^{\circ}$ ) have been excluded from major TAVR clinical trials. Therefore, available data on TAVR with Evolut in this challenging anatomy are limited, and standardized treatment strategies and clinical results remain unknown. Herein, we report a clinical case series of TAVR with Evolut in extremely horizontal aorta. Among seven patients (aged 80-92 years; STS score, 12.6\% $\pm 7.9 \%$ ) who underwent TAVR with Evolut system, aortic root angle ranged from $71^{\circ}$ to $83^{\circ}$ (mean, $75.1^{\circ} \pm 4.5^{\circ}$ ). All patients achieved device success with dedicated strategies and were clinically stable at 3-month follow-up. None of the patients had more than mild paravalvular leakage (PVL) at any point during follow-up.

Complications in three patients included complete atrioventricular block requiring a permanent pacemaker implantation, cerebral infarction because of atrial fibrillation 3 days after TAVR, and cardiac tamponade requiring pericardiocentesis. In this case series, Evolut self-expanding TAVR in extremely horizontal aorta was effective and feasible with a high device success rate. Based on anatomical features, some dedicated strategies majorly contribute to the success of this procedure. Large-scale multicenter studies are required to confirm our findings.
\end{abstract}

Key words: Aortic stenosis, Horizontal aortic root, Self-expanding valve

(Int Heart J 2020; 61: 1059-1069)

$\mathrm{T}$ ranscatheter aortic valve replacement (TAVR) with Evolut $\mathrm{R}^{\mathrm{TM}}$ or Evolut $\mathrm{PRO}^{\mathrm{TM}}$ self-expanding bioprosthesis (Medtronic, Minneapolis, Minnesota, USA) has been shown to be effective in treating severe aortic stenosis (AS) with relatively low rates of annular rupture, vascular complication, and long-term complications including paravalvular leakage (PVL). ${ }^{1-5)}$ However, because of its rigidity and non-steerability, the presence of a horizontal aortic root during TAVR with Evolut system can be problematic. According to prior studies, horizontal aortic root anatomy poses many difficulties in coaxial implantation of the self-expanding valve, resulting in increased fluoroscopy time, valve migration, aortic injury, need for a second valve, left ventricular perforation, ${ }^{6}$ postdilation, and postprocedural PVL. ${ }^{7)}$ For these reasons, most patients with extremely horizontal aorta (aortic root angle $\geq 70^{\circ}$ ) had been excluded from major TAVR clinical trials, ${ }^{8,9)}$ and available data in this complex anatomy have been limited to sporadic single-case reports. ${ }^{10,11)}$ Hence, the clinical results and standardized procedural strategy for TAVR with Evolut system remain unknown. Herein, we report the result of our clinical case series, which includes patients with extremely horizontal aorta who received TAVR with Evolut system. In addition to the conventional implantation technique, we developed two novel modified implantation protocols to facilitate coaxial valve implantation. This article aims to describe the important anatomical problems and major technical considerations with using Evolut systems and to finally establish dedicated strategies for treating patients with challenging anatomy.

From the ${ }^{1}$ Department of Cardiovascular Medicine, Sapporo Cardio Vascular Clinic, Sapporo Heart Center, Sapporo, Japan, ${ }^{2}$ Department of Cardiovascular Surgery, Sapporo Cardio Vascular Clinic, Sapporo Heart Center, Sapporo, Japan and ${ }^{3}$ Department of Anesthesiology, Sapporo Cardio Vascular Clinic, Sapporo Heart Center, Sapporo, Japan.

Address for correspondence: Umihiko Kaneko, MD, Department of Cardiovascular Medicine, Sapporo Cardio Vascular Clinic, Sapporo Heart Center, North 49, East 16, 8-1 Higashi Ward, Sapporo, Hokkaido 007-0849, Japan. E-mail: uk434471@gmail.com

Received for publication February 22, 2020. Revised and accepted June 9, 2020.

Released in advance online on J-STAGE September 12, 2020.

doi: 10.1536/ihj.20-120

All rights reserved by the International Heart Journal Association. 


\section{Case Report}

Between January 2017 and December 2019, 51 consecutive patients with severe symptomatic AS underwent TAVR, with Evolut R or Evolut PRO transcatheter heart valve, at Sapporo Cardio Vascular Clinic. We retrospectively assessed patients with extremely horizontal aorta who had undergone TAVR. Aortic root angle was evaluated by an expert radiologist using preprocedural multidetector computed tomography (MDCT). We defined extremely horizontal aorta as an aortic root angle $\geq 70^{\circ}$ between the horizontal plane and the plane of the aortic annulus in coronal projection. All patients were informed about the treatment strategy and gave informed consent. Baseline clinical, echocardiographic, and procedural data were recorded for all patients. In all patients, TAVR procedure was performed via either the transfemoral (TF) or left transaxillary (TAx) access after a multidisciplinary heart team discussion. All patients underwent an echocardiographic follow-up evaluation 1 day and 30 days after the procedure. Three months after the procedure, procedural characteristics and clinical outcome data were reported according to Valve Academic Research Consortium-2 (VARC-2) definition. ${ }^{12}$ Mortality was assessed at 3 months.

Among the 51 patients who underwent TAVR with Evolut, seven patients with extremely horizontal aorta (aged 80-92 years; STS score, $12.6 \% \pm 7.9 \%$ ) were reviewed. Aortic root angle ranged from $71^{\circ}$ to $83^{\circ}$ (mean, $\left.75.1^{\circ} \pm 4.5^{\circ}\right)$. Patients' descriptive data are shown in Table. Even in the presence of extremely horizontal aorta, Evolut self-expanding valve was selected as a better alternative for these patients, based on the following patient characteristic: presence of heavily calcified annulus, deemed as a high risk for annular rupture (Cases 2, 3, and 6 ), and tortuous descending aorta, shaggy aorta, or abdominal aortic aneurysm that resulted in femoral access being unsuitable, of which left TAx insertion was deemed the optimal access route (Cases 2, 4, 6, and 7); the small delivery profile of Evolut was required to overcome issues of problematic femoral access, specifically narrow iliac artery or tortuous descending aorta in Cases 1 and 5. Preprocedural CT assessment of annulus morphology, aortic root angle, and final angiographic images of each case are shown in Figure 1. Three cases were performed via TF access and the other four cases via left TAx access. All patients underwent successful TAVR procedures. Four cases were successfully treated with conventional implantation technique, two cases with modified technique 1, and one case with modified technique 2. Mean fluoroscopy time, radiation dose, and amount of contrast were $31.1 \pm 8.0$ minutes, $523 \pm 234 \mathrm{mGy}$, and $69.9 \pm 24.5 \mathrm{~mL}$, respectively. None of the patients required post-dilatation. No valve migration or embolization occurred. Complications were observed in three patients: complete atrioventricular block requiring implantation of a permanent pacemaker (Case 4); development of atrial fibrillation resulting in cerebral infarction, despite no postoperative neurological deficits, 3 days after TAVR (Case 7); and cardiac tamponade requiring pericardiocentesis (Case 2). No other major complications were noted. All patients achieved de- vice success (defined by VARC-2 guidelines) and were clinically stable at 3-month follow-up. None of the patients had more than mild PVL at any point during follow-up.

Procedural details in selected cases of TAVR with Evolut self-expanding valve in extremely horizontal aorta: Case 1 (treated with conventional technique) An 86-yearold man was referred to our hospital with symptomatic severe AS. Preprocedural CT revealed a 73.9-mm annulus with extremely horizontal aorta (aortic root angle, $77^{\circ}$ ) (Figure 2A). Because of narrow iliac artery and high risk of surgery, he received implantation of a 29-mm Evolut PRO via the right femoral artery. After placing the Safari Small wire (Boston Scientific, Marlborough, Massachusetts, USA) into the left ventricular apex along the interventricular septum (Figure $2 \mathrm{~B}$ and $\mathrm{C}$ ), we intentionally advanced the delivery catheter along the greater curvature of the ascending aorta (Figure 2D) and implanted the bioprosthesis while applying gentle forward force on the wire to facilitate "self-centering" of the device. When annular contact occurred from the noncoronary cusp (NCC) to the left coronary cusp (LCC), coaxial alignment between the bioprosthesis and the native aortic valve was not perfectly achieved (Figure 2E). Therefore, we adjusted the alignment by pushing the delivery catheter to achieve a coaxial alignment. The final angiogram showed satisfactory coaxial position of the bioprosthesis with trace PVL and mean pressure gradient (PG) of $5.2 \mathrm{mmHg}$ (Figure 2F).

Case 2 (treated with modified technique 1) An 89-year-old woman with symptomatic severe AS, severe aortic regurgitation, and significantly small left ventricular outflow tract (LVOT) was referred for TAVR, due to high risk of surgery. Preprocedural CT demonstrated significant tortuous descending aorta, type I aortic arch, and extremely horizontal aorta (aortic root angle, $83^{\circ}$ ) with high angulation (Figure 3A). In addition, heavily calcified annulus, septal hypertrophy, and LVOT (perimeter, $65.2 \mathrm{~mm}$ ) that was significantly smaller than the annulus (perimeter, 75.2 $\mathrm{mm}$ ) were noted. Considering unsuitable femoral access and high risk of annular rupture, our heart team decided that left TAx TAVR using a 29-mm Evolut R was a better treatment option instead of using a balloon-expandable valve. Advancing the Safari Small wire into the left ventricular apex posed major difficulties because of septal hypertrophy and the unfavorable alignment between the aorta and the left ventricle (LV). ${ }^{6}$ Despite several attempts, the stiff wire was located along the left ventricular lateral wall, away from the left ventricular septum and apex (Figure 3B, white arrowheads). After the advancement of the delivery catheter system along the greater curvature of the ascending aorta, the bioprosthesis was deeply deployed on the side of the NCC (Figure 3C and D). Therefore, we altered the treatment strategy and delivered the catheter along the lesser curvature of the ascending aorta while pulling back the guidewire (Figure 3E). This maneuver enabled us to achieve coaxial position (Figure 3F). However, during deployment, the bioprosthesis was dislodged from the annular ring because of small LVOT, resulting in the perpendicular stabbing of the NCC (Figure 3G, yellow arrowhead). Finally, because of rapid pacing $(130 \mathrm{bpm})$ and blood pressure management $(70 / 45$ 


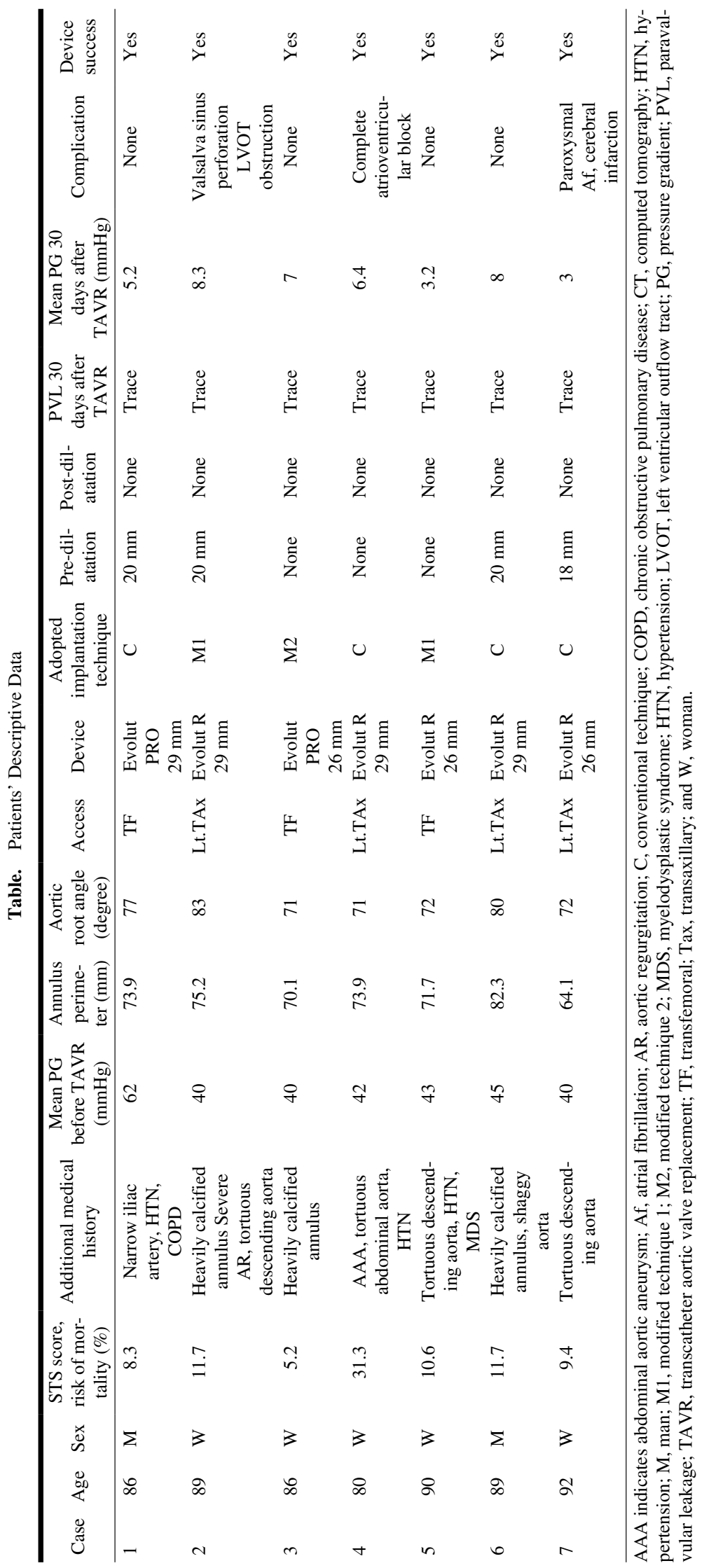



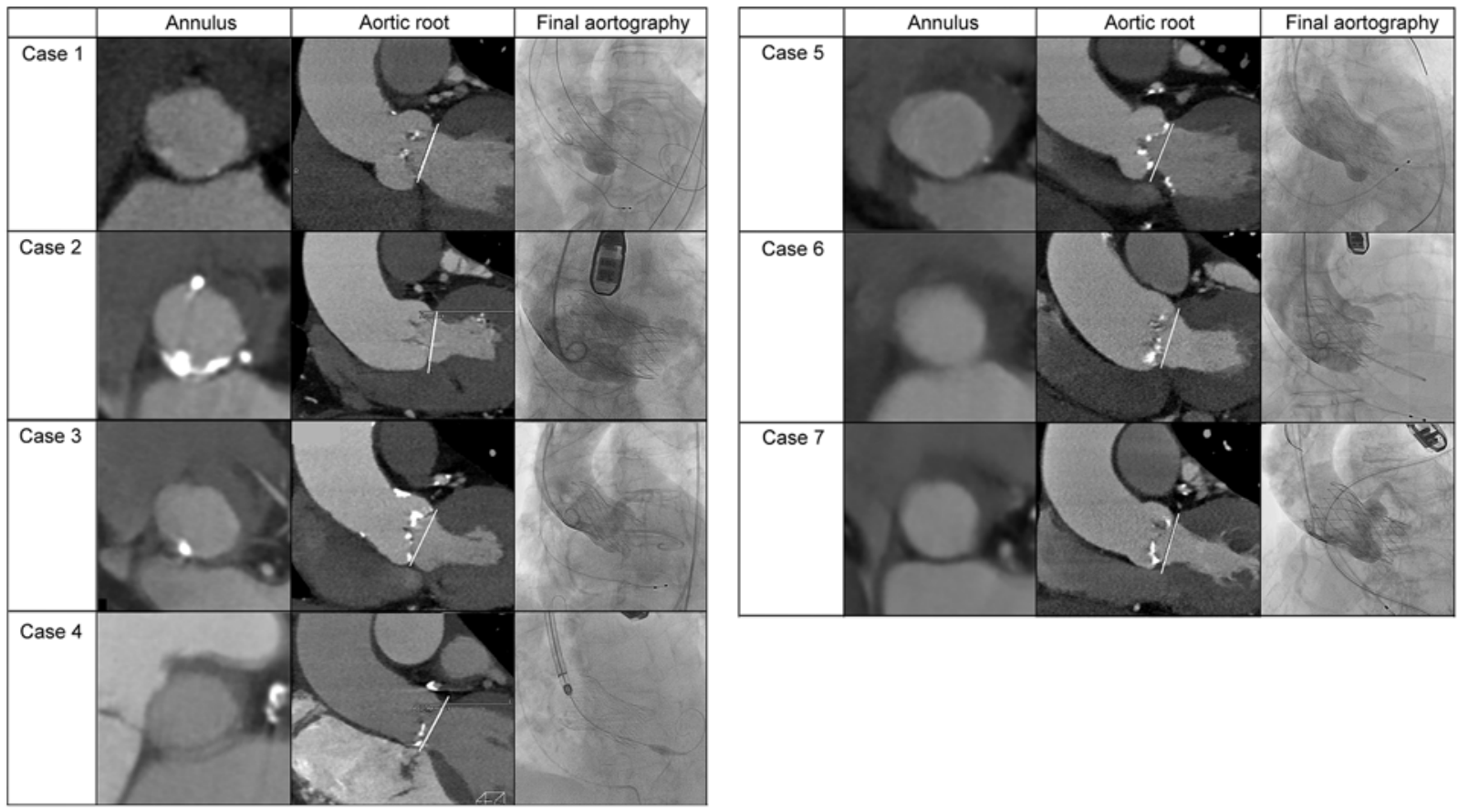

Figure 1. Preprocedural CT assessment of annulus morphology, aortic root angle, and final angiographic images of each case.
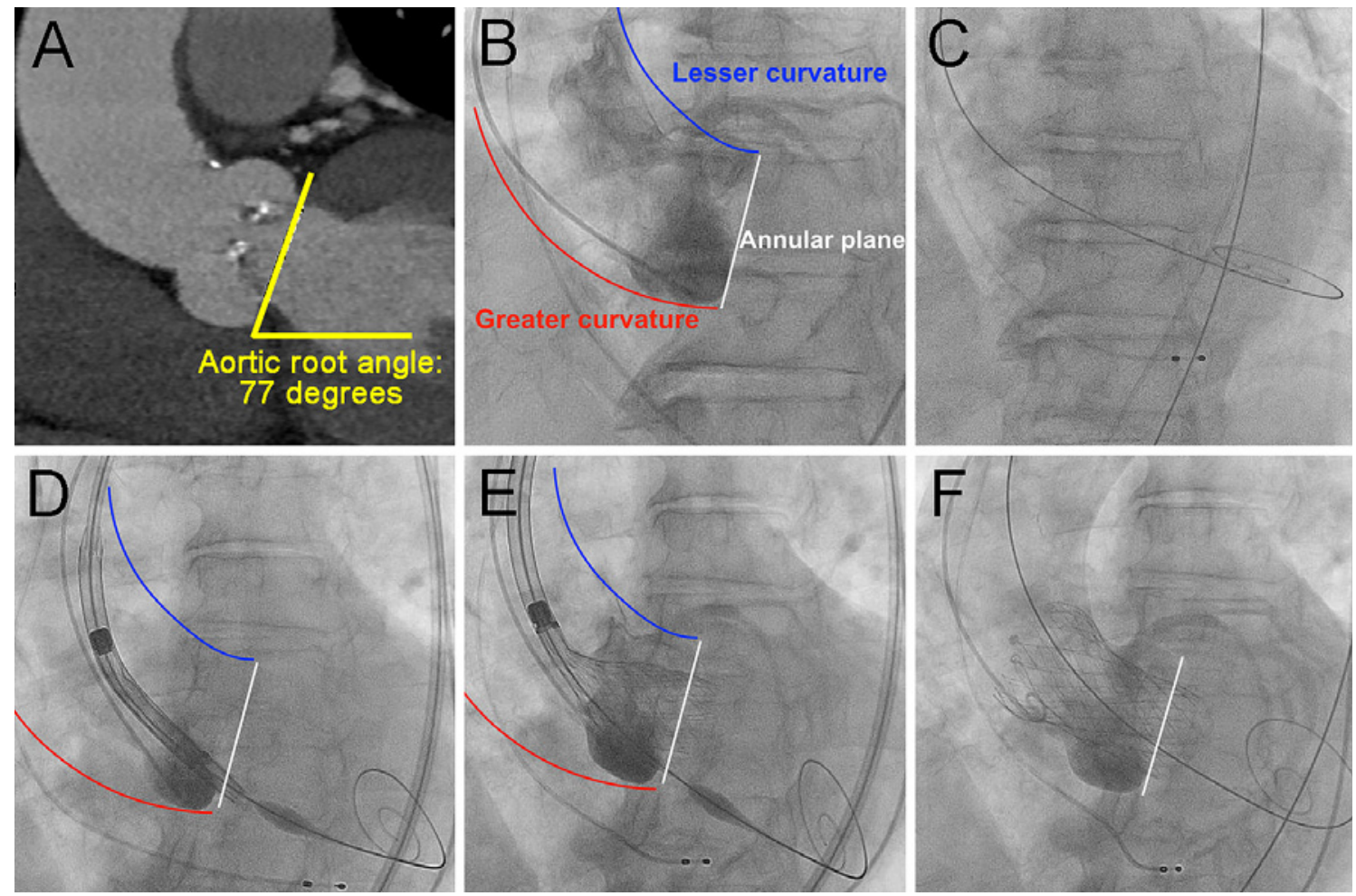

Figure 2. Preprocedural CT and angiographic images of Case 1 treated with the conventional technique. A: Preprocedural CT revealed extremely horizontal aorta (aortic root angle, $77^{\circ}$ ). B: Baseline aortography. C: The stiff wire was placed into the left ventricular apex along the interventricular septum. D: Advancement of the delivery catheter along the greater curvature of the aorta. E: Valve implantation while applying gentle forward force on the stiff wire. F: Final aortography showed satisfactory coaxial position. White line indicates the position of annular plane, blue line indicates the lesser curvature of the ascending aorta, and red line indicates the greater curvature. 

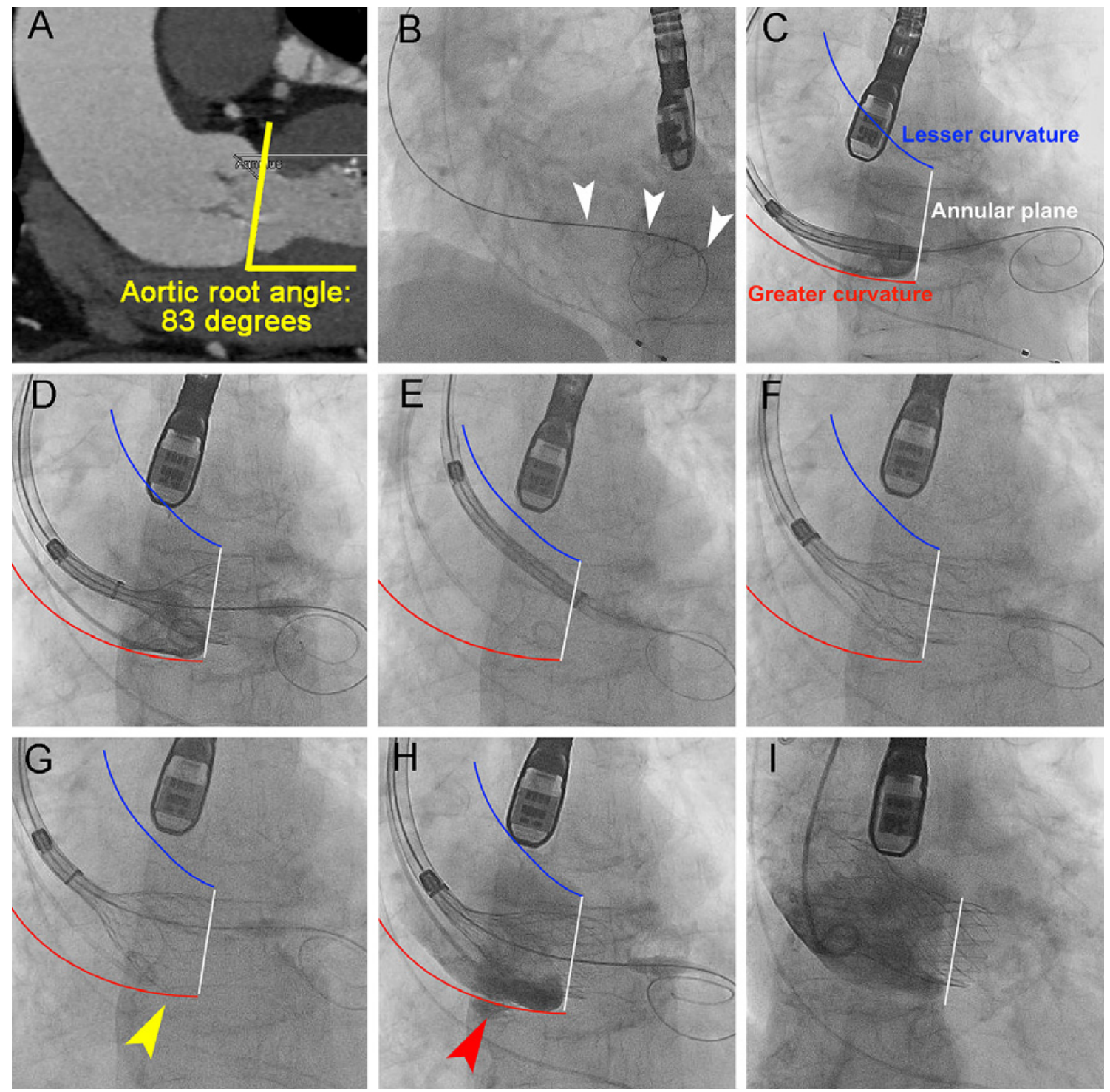

Figure 3. Preprocedural $\mathrm{CT}$ and angiographic images of Case 2 treated with modified technique 1. A: Preprocedural CT revealed extremely horizontal aorta (aortic root angle, $83^{\circ}$ ). B: The stiff wire was placed along the left ventricular lateral wall (white arrowheads). C and D: After advancing the delivery catheter system along the greater curvature of the aorta, the bioprosthesis was deeply deployed on the side of the noncoronary cusp (NCC). $\mathbf{E}$ and $\mathbf{F}$ : Advancement of the delivery catheter along the lesser curvature of the aorta while pulling back the guidewire enabled proper coaxial positioning. G: During deployment, the prosthesis was dislodged, and it perpendicularly stabbed the NCC (yellow arrowhead). H: Aortography demonstrated coalignment position of the prosthesis and Valsalva sinus perforation (red arrowhead). I: The final angiogram confirmed good coaxial position of the prosthesis without contrast extravasation at the NCC. White line indicates the position of annular plane, blue line indicates the lesser curvature of the ascending aorta, and red line indicates the greater curvature of the ascending aorta.

$\mathrm{mmHg}$ ), the bioprosthesis was successfully coaligned and deployed at a stable position without dislodgement. Aortography demonstrated Valsalva sinus perforation, which resulted from the stabbing by the inflow part of the dislodged prosthesis (Figure 3H, red arrowhead). Cardiac tamponade occurred but was successfully treated with pericardiocentesis. ${ }^{13)}$ The final angiogram showed good coaxial position of the bioprosthesis with an implantation depth of $4 \mathrm{~mm}$, trace PVL, and mean PG of $8.3 \mathrm{mmHg}$. No contrast extravasation at the NCC was confirmed (Figure 3I). At her 6-month follow-up, the patient remained stable and asymptomatic.

Case 3 (treated with modified technique 2) An 86-year-old woman with symptomatic severe AS underwent TF TAVR with a 26-mm Evolut PRO. Preprocedural CT and aortography demonstrated extremely horizontal (root angle, $71^{\circ}$ ), angulated aortic root with heavy annular calcification (Figure 4A). Because of the presence of heavy annular calcification, Evolut bioprosthesis was deemed the most suitable device to minimize the risk of annular rupture. At first, the Safari Extra Small wire was inserted inversely in the LV, i.e., positioned along the left ventricular 

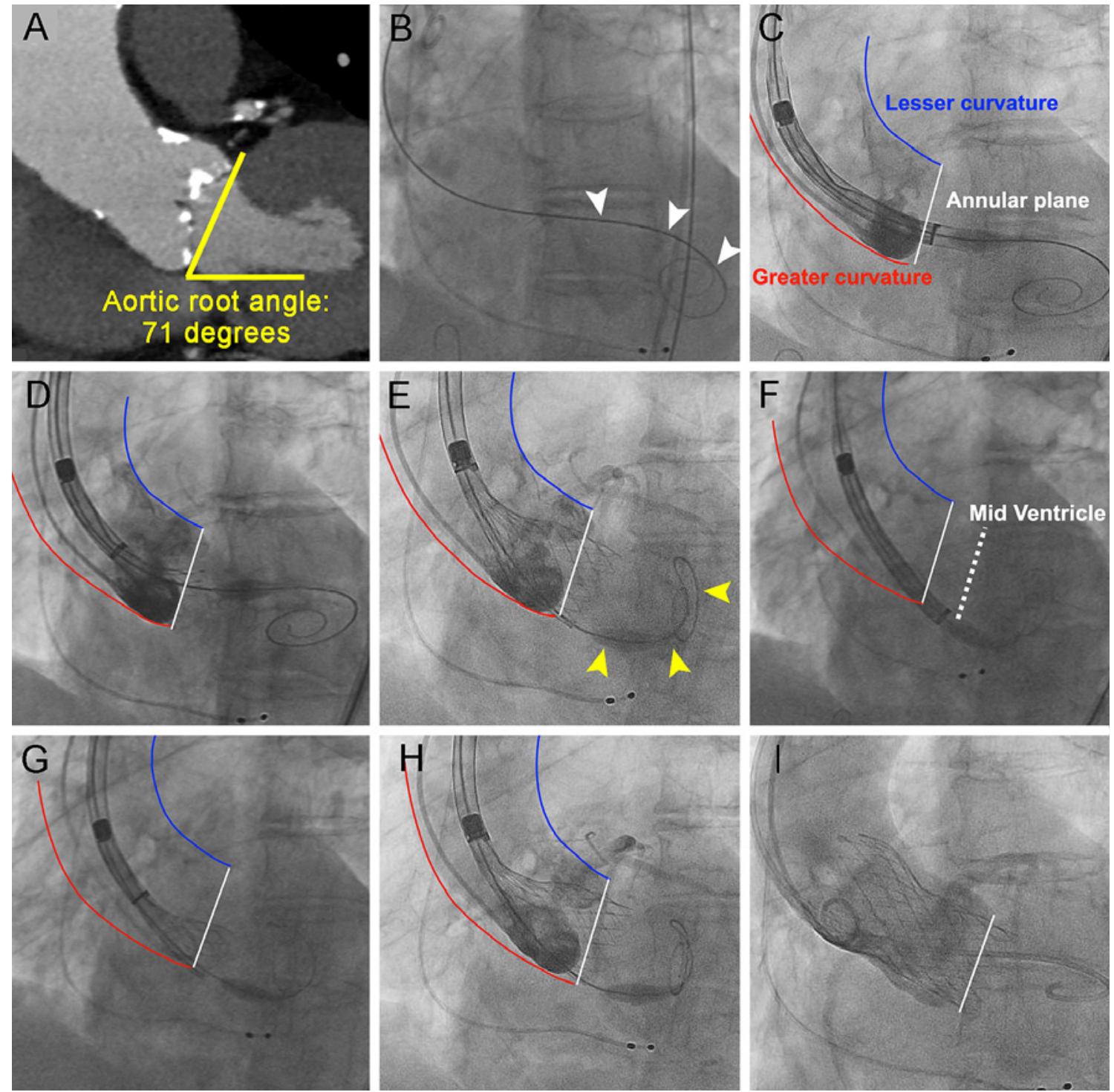

Figure 4. Preprocedural CT and angiographic images of Case 3 treated with modified technique 2. A: Preprocedural CT revealed extremely horizontal aorta (aortic root angle, $71^{\circ}$ ). B: The stiff wire was positioned along the left ventricular lateral wall (white arrowheads). C and D: Advancement of the delivery catheter along the greater curvature of the ascending aorta made the bioprosthesis deeply deployed on the side of the noncoronary cusp. E: Although the guidewire was repositioned along the interventricular septum (yellow arrowheads), the bioprosthesis was deeply deployed on the side of the left coronary cusp. F: Inserting the delivery catheter deeply into the mid-ventricle. G: Pulling up the delivery catheter during valve deployment and adjusting the final implantation depth. H: These maneuvers allowed a coaxial deployment. I: The final angiogram showed coaxial valve position with an implantation depth of $4 \mathrm{~mm}$. White line indicates the position of annular plane, white dotted line indicates the estimated position of mid-ventricle, blue line indicates the lesser curvature of the ascending aorta, and red line indicates the greater curvature of the ascending aorta.

lateral wall, because of extremely horizontal aorta and unfavorable alignment between the aorta and the LV (Figure 4B). After the advancement of the delivery catheter system along the greater curvature of the ascending aorta (Figure 4C), the valve was deeply deployed on the NCC side, which required recapturing (Figure 4D). After recapture and guidewire handling, the guidewire was repositioned along the interventricular septum. Despite appropriate guidewire position, the bioprosthesis was deeply deployed on the side of the LCC (Figure 4E). To establish coaxial valve positioning, after inserting the delivery catheter deeply into the mid-ventricle (Figure 4F), we gradually pulled up the delivery catheter during valve deployment and finally adjusted the implantation depth (Figure $4 \mathrm{G}$ and $\mathrm{H}$ ). These maneuvers allowed a coaxial deployment. Finally, the valve was successfully deployed in coaxial position with only trace PVL and a mean PG of $7.0 \mathrm{mmHg}$ (Figure 4I).

Case 4 (treated with conventional technique via left TAx access in type III aortic arch) An 81-year-old woman with symptomatic severe AS received implantation of a 29-mm Evolut R via left TAx access due to abdominal 

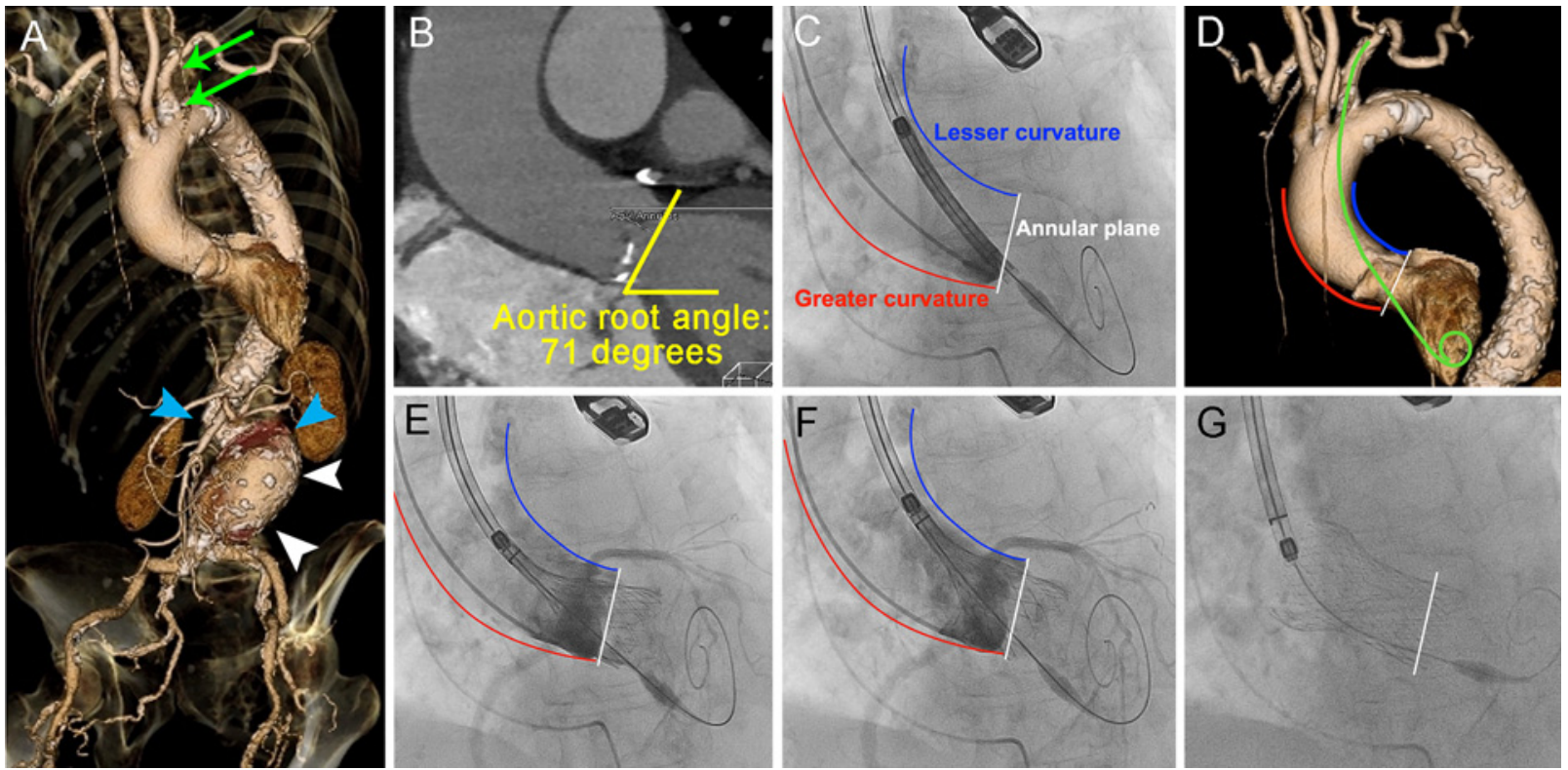

Figure 5. Preprocedural CT and angiographic images of Case 4 treated with conventional technique via left transaxillary access in type III aortic arch. A: Three-dimensional CT reconstruction of the left anterior oblique projection revealed type III aortic arch in which the left subclavian artery originated low and proximal on the aortic arch (green arrows). Abdominal aortic aneurysm (white arrowheads) and significantly tortuous abdominal aorta (blue arrowheads) were found. B: Preprocedural CT demonstrated extremely horizontal aorta (aortic root angle, $71^{\circ}$ ). C: Both the stiff wire and the delivery catheter were unfavorably positioned toward the lesser curvature of the ascending aorta. D: Preprocedural CT on that position of the stiff wire projected revealed specific vessel anatomy of aortic arch type III and left subclavian artery. Green line indicates the course of the stiff wire going along the lesser curvature of the ascending aorta. E: Due to the horizontal shape, the prosthesis was persistently lower on the left sinus. F: Angiography showed satisfactory depth on the noncoronary sinus. G: The final fluoroscopic image showed good coaxial position of the bioprosthesis with an implantation depth of $5 \mathrm{~mm}$. White line indicates the position of annular plane, blue line indicates the lesser curvature of the ascending aorta, and red line indicates the greater curvature of the ascending aorta.

aortic aneurysm and significantly tortuous abdominal aorta (Figure 5A). Preprocedural CT revealed a 73.9-mm annulus with extremely horizontal (root angle, $71^{\circ}$ ) and angulated aortic root (Figure 5B). Preprocedural CT revealed type III aortic arch in which the left subclavian artery originated low and proximal on the aortic arch (Figure 5 A, green arrows). Owing to this specific vessel anatomy, both the small Safari guidewire and the delivery catheter were inappropriately positioned toward the lesser curvature of the ascending aorta, resulting in misalignment (Figure 5C and D). Therefore, we initiated valve deployment from a higher position in NCC (i.e., $2 \mathrm{~mm}$ below the annulus). However, the valve was deployed deeply on the LCC side due to the horizontal shape (Figure 5E). After recapture and several attempts, we started deployment at $0-\mathrm{mm}$ position of NCC while applying gentle forward force on the wire to facilitate the device to self-center. Once the valve engaged the left side, we adjusted the alignment by pushing the delivery catheter (Figure 5F). The final fluoroscopic image showed good coaxial position of the bioprosthesis with trace PVL and an implantation depth of $5 \mathrm{~mm}$ (Figure 5G). After the TAVR procedure, the patient developed complete atrioventricular block, requiring permanent pacemaker implantation.

\section{Discussion}

This is the first study describing procedural strategies and clinical outcomes of TAVR using Evolut self- expanding valve in patients with extremely horizontal aorta. This study presents two important findings. First, TAVR with Evolut self-expanding valve in extremely horizontal aorta was effective and feasible with high device success rate, trace/mild PVL, and no 3-month mortality. Second, based on MDCT assessment and patient anatomy, extremely horizontal aortic root requires specific technical considerations, including two novel modified techniques we developed.

According to the recent evidence, Evolut bioprosthesis proved to have similar clinical outcomes when compared to SAPIEN 3 balloon-expandable valves (Edwards Lifesciences Inc., Irvine, California, USA). ${ }^{3,14)}$ In patients with horizontal aorta, the SAPIEN 3 delivery system provides the "distal flex" that enables controlled coaxial alignment. Meanwhile, the Evolut system and firstgeneration self-expanding devices have the drawback of rigidity and non-steerability. Further, the use of firstgeneration self-expanding devices was associated with increased complication rates and lower device success rates in patients with horizontal aorta. ${ }^{7}$ Recently, some studies reported that the presence of horizontal aorta has no impact on device success nor on short-term clinical outcomes of TAVR with second-generation self-expanding devices. ${ }^{15,16)}$ However, most patients with extremely horizontal aorta had been excluded from major studies. ${ }^{8.9}$ The instructions for the use of Evolut prohibit its use via TF or left TAx access in the presence of extremely horizontal aorta because of its rigidity and non-steerability. The re- 
port of TAVR in this specific subset has been scarce, and standardized treatment strategies and clinical results remain uncertain.

However, Evolut system has a number of advantages, including the small delivery profile compared to other TAVR devices; supra-annular valve design, which promotes single-digit gradients and large effective orifice areas; self-expanding frame, which lowers the risk of annular rupture; and the ability to recapture and reposition. Therefore, even in the presence of extremely horizontal aorta, the use of Evolut system is frequently required for many complex anatomical subsets such as poor access route, heavily calcified annulus, small annulus, bicuspid aortic valves, and degenerated surgical valve. Horizontal aortic root has been one of the most important anatomical problems to be overcome during TAVR with Evolut system.

Evolut self-expanding TAVR in extremely horizontal aortic root poses two great challenges: 1) aortic valve crossing and 2) coaxial deployment. Regarding aortic valve crossing in patients with extremely horizontal aortic root, the ascending aorta tends to be severely angulated and elongated (Figure 1). Therefore, tracking the delivery catheter along the ascending aorta to the annulus and aortic valve crossing are associated with significant technical challenges. Because of the rigidity and non-steerability, the delivery catheter tends to be advanced along the greater curvature of the ascending aorta. When the advancement of the delivery catheter through the angulated aorta fails, the edge of the delivery catheter tends to scrape the aortic wall, leading to aortic injury. A recent study reported a high incidence rate of fatal complications, such as aortic dissection and aortic or annular rupture, among individuals with a horizontal aorta, ${ }^{17)}$ warranting careful procedural planning when this complex anatomy is encountered.

To avoid catastrophic aortic injury and successfully advance the delivery catheter into the LV, the following strategies can be employed: the coordinated manipulation of the guidewire and the delivery system; the use of a stiffer guidewire, such as Lunderquist Extra-Stiff Wire Guide (Cook Inc., USA); ${ }^{11)}$ the employment of the buddy wire technique; and the use of the snare technique to allow greater detachment from the greater curvature of the ascending aorta, allowing the delivery catheter to be safely advanced. ${ }^{18)}$

Regarding coaxial deployment, the achievement of coaxial alignment between transcatheter bioprosthesis and native aortic valve poses major difficulties. The difficulty varies depending on the position of both the stiff wire in the LV and the transcatheter bioprosthesis in the aortic root. The choice, management, and position of the stiff wire in LV is of utmost importance especially in this setting.

To facilitate coaxial deployment, TAVR in extremely horizontal aortic root requires three dedicated implantation techniques including one conventional technique and two modified techniques described below. Figure 6 presents comparisons of the three dedicated implantation techniques.

The conventional implantation techniques include placing of the stiff wire in the left ventricular apex along the left ventricular septum (Figure 6A); advancing the delivery catheter toward the greater curvature of the ascending aorta (Figure 6B); stabilizing the delivery catheter by applying gentle forward force on the stiff wire during deployment to avoid deep implantation; and adjusting the alignment by pushing the delivery catheter by applying gentle forward force after the valve is engaged to left side (Figure 6C). ${ }^{9}$ Evolut bioprosthesis has a nitinol delivery catheter capsule that facilitates "self-centering" during release, leading to more symmetrical annular contact and coaxial deployment. These new self-expanding designs that allow self-centering simplify the implantation procedure in the presence of extremely horizontal aorta. Even if coaxial alignment between the bioprosthesis and the native aortic valve is not perfectly achieved after annular contact occurs between the NCC to LCC, the final alignment adjustment by pushing the delivery catheter can achieve a coaxial alignment (Figure 5F and 5G).

During the final phase of valve deployment, some anatomical and technical considerations need to be taken into consideration, including aortic angulation, angle between the delivery catheter and the aortic annulus, and the interaction between the upper stent frame and the ascending aorta. ${ }^{19)}$ Two recent studies demonstrated that displacement or micro-dislodgement of the Evolut bioprosthesis during the final release can occur under specific conditions. One study revealed that the angle between the bioprosthesis and the ascending aorta, at the point of recapture, is strongly associated with upward displacement of bioprosthesis on the LCC side. ${ }^{20)}$ The other study reported that micro-dislodgement was observed in nearly one-third of patients and that a larger aortic valve area was specified as a risk factor for micro-dislodgement. ${ }^{21)}$ Based on these reports, operators should pay special attention to risk factors (aorta/annulus angle or larger valve area) that can influence the behavior of the bioprosthesis during the final release.

In patients with extremely horizontal aorta, the stiff wire tends to be inserted along the left ventricular lateral wall (Figures 3B, 4B) because of anatomical misalignment between the axis of ascending aorta and the LV. ${ }^{6}$ According to previous study, the advancement of the delivery catheter along the greater curvature of the ascending aorta and the application of forward tension on the stiff wire have been recommended as the optimal technique in horizontal aorta." However, sometimes, this maneuver promotes deep deployment of the bioprosthesis on the NCC side, resulting in misalignment of position in instances where the stiff wire is positioned along the left ventricular lateral wall (Figures 4D, 6E).

By contrast, in instances where the stiff wire is located along the interventricular septum, the bioprosthesis tends to be deeply deployed on LCC side due to extremely horizontal anatomy (Figures 4E, 5E).

The most critical problem in these situations is the misalignment of the stiff wire and the delivery catheter between the ascending aorta, basal annular plane, and LV, leading to unfavorable valve deployment and misalignment with the central axis of the aortic annulus (Figure 6 $\mathrm{E}$ and $6 \mathrm{I})$. This misplacement can lead to non-coaxial 


\section{Conventional Technique}
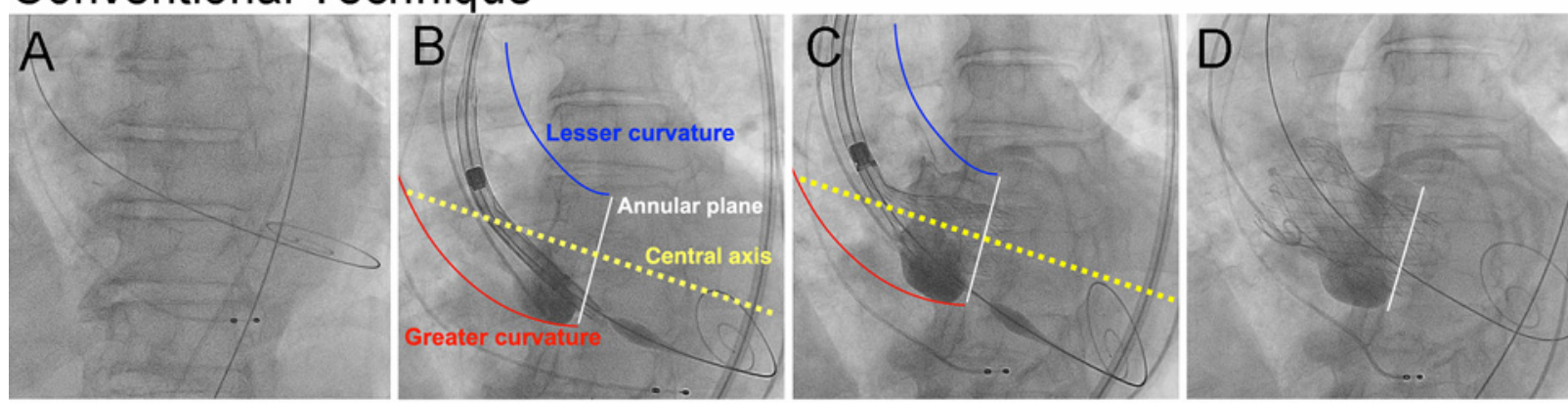

\section{Modified Technique 1}
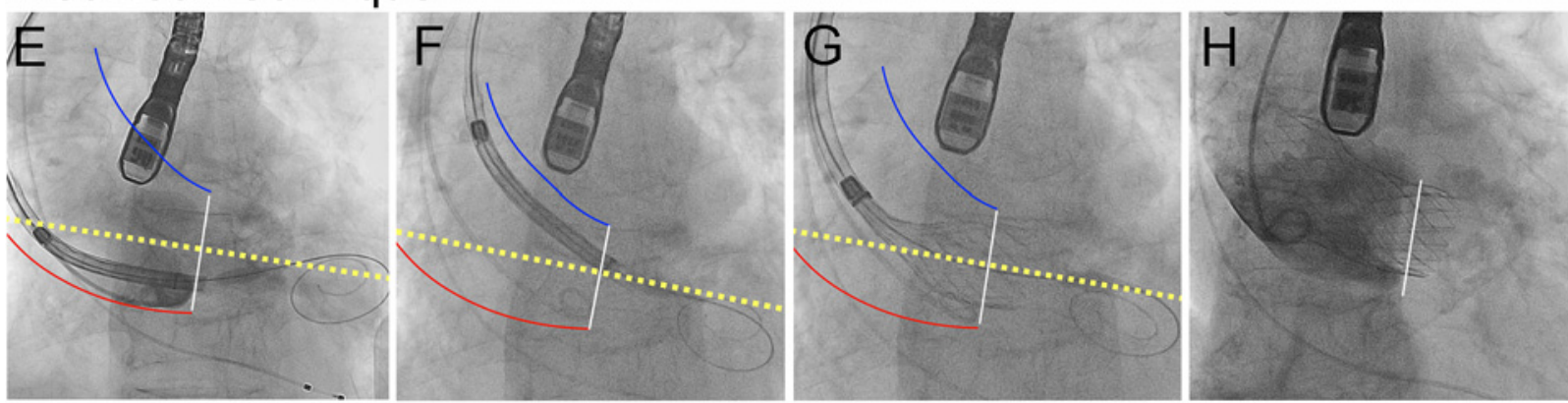

\section{Modified Technique 2}
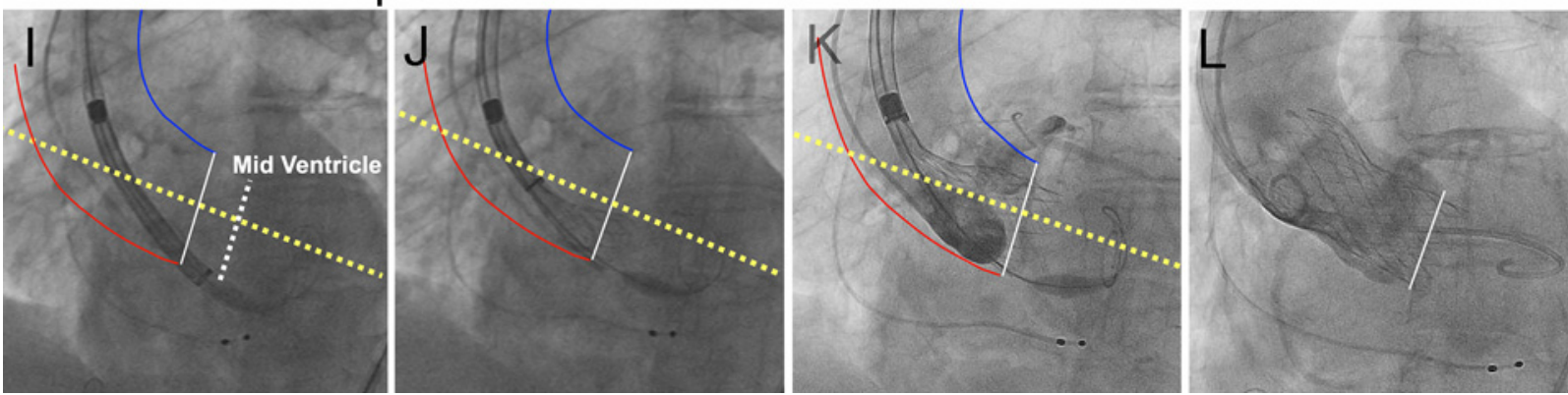

Figure 6. Comparison of three dedicated implantation techniques in extremely horizontal aorta. A-D: (Conventional technique) After the stiff wire was placed into the left ventricular apex along the interventricular septum, advancement of the delivery catheter along the greater curvature of the aorta and implantation of the prosthesis was successful. E-H: (Modified technique 1) Because the stiff wire was located along the left ventricular lateral wall, advancement of the delivery catheter along the greater curvature of the ascending aorta made the bioprosthesis deeply deployed on the side of the noncoronary cusp (E). We advanced the delivery catheter along the lesser curvature of the ascending aorta while pulling back the guidewire, leading to improvement of the unfavorable alignment between the bioprosthesis and the central axis of the aortic annulus. The final angiogram showed coaxial valve position (F-H). I-L: (Modified technique 2) After inserting the delivery catheter into the mid-ventricle, we pulled up the delivery catheter during valve deployment and adjusted implantation depth. Finally, the valve was successfully deployed in coaxial position. White line indicates the position of annular plane, blue line indicates the lesser curvature of the ascending aorta, red line indicates the greater curvature of the ascending aorta, yellow dotted line indicates the central axis of aortic annulus, and white dotted line indicates the estimated position of the mid-ventricle.

valve position, significant postprocedural PVL, or valve migration. ${ }^{7)}$ To specifically address this concern, we developed two modified techniques described as follows:

Modified technique 1) If the stiff wire is positioned along the left ventricular lateral wall and the bioprosthesis is deployed deeply on the NCC side at first attempt, advancing the delivery catheter intentionally along the lesser curvature of the ascending aorta while pulling back the stiff wire will promote proper alignment (Figure $6 \mathrm{~F}$ and 6 $\mathrm{G) \text {. }}$
Modified technique 2) If the stiff wire is located along the interventricular septum and the bioprosthesis is deployed deeply on the LCC side at first attempt, the delivery catheter must first be inserted into the midventricle; then, the delivery catheter must be pulled up during valve deployment, and finally, the implantation depth must be adjusted (Figure $6 \mathrm{~J}$ and $6 \mathrm{~K}$ ).

These two novel techniques can prevent the misalignment between the ascending aorta and the LV, finally resulting in coaxial valve deployment with proper alignment 
to the central axis of the aortic annulus (Figure $6 \mathrm{H}$ and 6 L).

Depending on the position of both the stiff wire in the LV and the delivery catheter in the aortic root, we can choose the best implantation techniques to facilitate coaxial positioning and deployment of the bioprosthesis. Anticipation of difficulties and careful planning based on anatomical features and meticulous preprocedural CT analysis should be mandatory.

Regarding vascular access, recent studies confirm that, when the risk of femoral access is considered too high, TAx TAVR with Evolut bioprosthesis provides similar mortality with $\mathrm{TF}$ access and has become the most preferable alternative access route. ${ }^{22,23)}$ Generally, right TAx access should not be selected in patients with extremely horizontal aorta due to inability to achieve coaxial alignment of the bioprosthesis with the aortic root and annulus. To appropriately determine the viability of left TAx TAVR, preprocedural CT assessment of the aortic arch should be used to confirm the angulation and position of the takeoff of the left subclavian artery from the aortic arch. $^{24,25)}$ In type I aortic arch where the origin of all three major arteries are located in the same horizontal plane as the greater curvature of the aortic arch, the stiff wire and the delivery catheter can be efficiently advanced along the greater curvature of the ascending aorta. In this respect, the left TAx access should be selected preferentially since it closely mimics TF access. Some authors have suggested that, compared with transaortic or transapical access, which provide prosthesis malalignment due to the short distance between the delivery catheter and the aortic annulus, the left TAx access may allow the bioprosthesis to conform better to the greater curvature of the ascending aorta. ${ }^{26)}$ When compared with the TF access, the left TAx access can facilitate precise positioning by improving the control of the bioprosthesis during deployment because of the very short distance between the access site and the aortic annulus, leading to the reduction of the incidence of PVL and complete atrioventricular block. ${ }^{27)}$

In contrast, type III aortic arch, in which the left subclavian artery originates low and proximal on the aortic arch, can be very challenging for left TAx TAVR, requiring specific considerations and choice of the stiff wire (Figure 5A and 5D). In a patient with type III aortic arch or some needing TF access, both the stiff wire and the delivery catheter tend to be unfavorably advanced along the lesser curvature of the ascending aorta, making the bioprosthesis persistently deeper on LCC side, leading to a misaligned position (Figures 4E, 5E). In this complex subset, valve deployment should be started from higher position in NCC (i.e., 0-2 mm below the annulus). Applying gentle forward force on the stiff wire to facilitate "selfcentering" of the device is also required. Furthermore, the use of a Double Curved Lunderquist Extra-Stiff Wire Guide (Cook Medical, Bloomington, Indiana, USA) is a possible solution for achieving coaxial deployment. This stiff wire has a characteristic second curve that can be preferably positioned along the greater curvature of the ascending aorta. This particular configuration of the wire overcomes the steep angle, allowing the delivery catheter to conform better to the greater curvature of the ascending aorta needed. Another important point in favor of this guidewire is its stiffness. The stiffness of this wire is more than twofold that of other stiff wires and allows for more stable implantations, providing better control during valve deployment in patients with horizontal aorta or extensive tortuous aortic root. ${ }^{7,20)}$

To the best of our knowledge, this is the first report describing the clinical outcomes and technical aspects of Evolut self-expanding TAVR in patients with extremely horizontal aorta. In this case series, TAVR with Evolut prosthesis was effective and feasible with a high device success rate. This was only achieved through the assessment of several anatomical features and careful preprocedural planning with CT assessment. Dedicated strategies play a pivotal role in the success of this procedure. Further large multicenter studies are required to confirm our findings regarding this complex anatomical subset.

There are some limitations in this study. First, this study is a single-center retrospective study with a small number of patients. Consequently, the generalization of the findings of this study may not be recommended. However, this is the first study describing technical aspects and clinical outcomes of TAVR with Evolut prosthesis in patients with extreme horizontal aorta. Some dedicated strategies, including the two modified techniques we have presented here, can be effective in this specific subset. Second, patient selection was left to physician's discretion. Third, dedicated core laboratory assessment of CT and echocardiographic findings are lacking in this study.

\section{Conclusion}

In this case series, TAVR with Evolut self-expanding valve in patients with extremely horizontal aorta was effective and feasible with high device success rate. The success of this procedure is based on careful consideration of several anatomical features and some dedicated strategies, including two novel modified techniques described here. Large-scale multicenter studies are required to confirm our findings regarding this challenging anatomy.

\section{Acknowledgment}

The authors thank all the members of the heart team at the Sapporo Cardio Vascular Clinic.

\section{Disclosure}

Conflicts of interest: All the authors declare that there are no conflicts of interest.

\section{References}

1. Popma JJ, Deeb GM, Yakubov SJ, et al. Transcatheter aorticvalve replacement with a self-expanding valve in low-risk patients. N Engl J Med 2019; 380: 1706-15.

2. Grube E, Van Mieghem NM, Bleiziffer S, et al. Clinical outcomes with a repositionable self-expanding transcatheter aortic valve prosthesis: the International FORWARD Study. J Am Coll Cardiol 2017; 70: 845-53.

3. Vlastra W, Chandrasekhar J, Muñoz-Garcia AJ, et al. Compari- 
son of balloon-expandable vs. self-expandable valves in patients undergoing transfemoral transcatheter aortic valve implantation: from the CENTER-collaboration. Eur Heart J 2019; 40: 456-65.

4. Popma JJ, Reardon MJ, Khabbaz K, et al. Early clinical outcomes after transcatheter aortic valve replacement using a novel self-expanding bioprosthesis in patients with severe aortic stenosis who are suboptimal for surgery: results of the Evolut R US Study. JACC Cardiovasc Interv 2017; 10: 268-75.

5. Tsuru Y, Miura M, Shirai S, et al. Aortic complex rupture after transcatheter aortic valve implantation. Int Heart J 2019; 60: 772-7.

6. Ancona MB, Hachinohe D, Giannini F, et al. Hypertrophic left ventricle with small cavity and severe aortic angulation: a dangerous association in case of transcatheter aortic valve replacement. JACC Cardiovasc Interv 2018; 11: e29-30.

7. Abramowitz Y, Maeno Y, Chakravarty T, et al. Aortic angulation attenuates procedural success following self-expandable but not balloon-expandable TAVR. JACC Cardiovasc Imaging 2016; 9: 964-72.

8. Adams DH, Popma JJ, Reardon MJ, et al. Transcatheter aorticvalve replacement with a self-expanding prosthesis. N Engl J Med 2014; 370: 1790-8.

9. Popma JJ, Reardon MJ, Yakubov SJ, et al. Safety and efficacy of self-expanding TAVR in patients with aortoventricular angulation. JACC Cardiovasc Imaging 2016; 9: 973-81.

10. Chan PH, Alegria-Barrero E, Di Mario C. Difficulties with horizontal aortic root in transcatheter aortic valve implantation. Catheter Cardiovasc Interv 2013; 81: 630-5.

11. Gunasekaran S, Ganesapandi R, Sivaprakasam MC. Transfemoral transcatheter aortic valve implantation in bicuspid aortic valve with extreme horizontal aorta to left ventricle angulation challenges. IHJ Cardiovasc CASE Rep (CVCR) 2018; 2: S54-6.

12. Kappetein AP, Head SJ, Généreux P, et al. Updated standardized endpoint definitions for transcatheter aortic valve implantation: The Valve Academic Research Consortium-2 consensus document. J Am Coll Cardiol 2012; 60: 1438-54.

13. Kaneko U, Kobayashi K, Hachinohe D, Fujita T. Valsalva sinus perforation following valve dislodgement during transcatheter aortic valve replacement. JACC Cardiovasc Interv 2019; 12: e119-20.

14. Thiele H, Kurz T, Feistritzer HJ, et al. Comparison of newer generation self-expandable vs. balloon-expandable valves in transcatheter aortic valve implantation: the randomized SOLVETAVI trial. Eur Heart J 2020; 41: 1890-9.

15. Di Stefano D, Colombo A, Mangieri A, et al. Impact of horizontal aorta on procedural and clinical outcomes in second- generation transcatheter aortic valve implantation. EuroIntervention 2019; 15: e749-56.

16. Bob-Manuel T, Pour-Ghaz I, Sharma A, et al. Correlation between aortic angulation and outcomes of transcatheter aortic valve replacement with new-generation valves. Curr Probl Cardiol 2019; 14: 100415.

17. Abdel-Wahab M, Kitamura M, Thiele H. TAVI and horizontal aorta: a "no impact" relationship? EuroIntervention 2019; 15: e736-8.

18. Medda M, Casilli F, Tespili M, Bande M. The "Chaperone Technique": valve-in-valve TAVR procedure with a self-expandable valve inside a degenerated sutureless prosthesis. JACC Cardiovasc Interv 2020; 13: e15-8.

19. Van Belle E, Delhaye C, Vincent F. The mirage of the optimal implantation depth with transcatheter bioprosthesis. JACC Cardiovasc Interv 2020; 13: 689-92.

20. Hachinohe D, Latib A, Laricchia A, et al. Anatomic and procedural associations of transcatheter heart valve displacement following Evolut R implantation. Catheter Cardiovasc Interv 2019; 93: $522-9$.

21. Hellhammer K, Piayda K, Afzal S, et al. Micro-dislodgement during transcatheter aortic valve implantation with a contemporary self-expandable prosthesis. PLOS ONE 2019; 14: $\mathrm{e} 0224815$.

22. Gleason TG, Schindler JT, Hagberg RC, et al. Subclavian/axillary access for self-expanding transcatheter aortic valve replacement renders equivalent outcomes as transfemoral. Ann Thorac Surg 2018; 105: 477-83.

23. Amat-Santos IJ, Rojas P, Gutiérrez H, et al. Transubclavian approach: A competitive access for transcatheter aortic valve implantation as compared to transfemoral. Catheter Cardiovasc Interv 2018; 92: 935-44.

24. Shen S, Jiang X, Dong H, et al. Effect of aortic arch type on technical indicators in patients undergoing carotid artery stenting. J Int Med Res 2019; 47: 682-8.

25. Nagpal H, Sharma PK, Chopra J, Patel R. Aortic Arch morphometry and its clinical implication -A computed tomography study. Arch Anat Physiol 2018; 3: 5-8.

26. Petronio AS, De Carlo M, Giannini C, De Caro F, Bortolotti U. Subclavian TAVI: more than an alternative Access route. EuroIntervention 2013; 9: S33-7.

27. Petronio AS, De Carlo M, Bedogni F, et al. Safety and efficacy of the subclavian approach for transcatheter aortic valve implantation with the CoreValve revalving system. Circ Cardiovasc Interv 2010; 3: 359-66. 